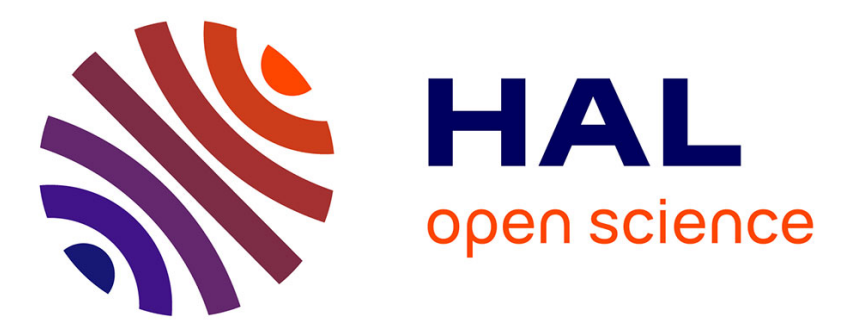

\title{
Ultrafast Synchrotron-Enhanced Thermalization of Laser-Driven Colliding Pair Plasmas
}

\author{
M. Lobet, C. Ruyer, A. Debayle, E. D'humières, M. Grech, M. Lemoine, L.
} Gremillet

\section{- To cite this version:}

M. Lobet, C. Ruyer, A. Debayle, E. D'humières, M. Grech, et al.. Ultrafast Synchrotron-Enhanced Thermalization of Laser-Driven Colliding Pair Plasmas. Physical Review Letters, 2015, 115 (21), 10.1103/PhysRevLett.115.215003 . hal-01821012

\section{HAL Id: hal-01821012 \\ https://hal.inria.fr/hal-01821012}

Submitted on 24 Nov 2021

HAL is a multi-disciplinary open access archive for the deposit and dissemination of scientific research documents, whether they are published or not. The documents may come from teaching and research institutions in France or abroad, or from public or private research centers.
L'archive ouverte pluridisciplinaire HAL, est destinée au dépôt et à la diffusion de documents scientifiques de niveau recherche, publiés ou non, émanant des établissements d'enseignement et de recherche français ou étrangers, des laboratoires publics ou privés.

\section{(c)(1)}

Distributed under a Creative Commons Attribution| 4.0 International License 


\title{
Ultrafast Synchrotron-Enhanced Thermalization of Laser-Driven Colliding Pair Plasmas
}

\author{
M. Lobet, ${ }^{1,2, *}$ C. Ruyer, ${ }^{1}$ A. Debayle, ${ }^{1}$ E. d'Humières,${ }^{2}$ M. Grech, ${ }^{3}$ M. Lemoine, ${ }^{4}$ and L. Gremillet ${ }^{1, \dagger}$ \\ ${ }^{1}$ CEA, DAM, DIF, F-91297, Arpajon, France \\ ${ }^{2}$ CELIA, UMR 5107, Université de Bordeaux-CNRS-CEA, 33405, Talence \\ ${ }^{3}$ LULI, UMR 7605, CNRS-CEA-École Polytechnique-Université Paris VI, École Polytechnique, 91128 Palaiseau, France \\ ${ }^{4}$ Institut d'Astrophysique de Paris, CNRS, UPMC, 98 bis boulevard Arago, F-75014 Paris, France \\ (Received 7 March 2014; revised manuscript received 25 August 2015; published 17 November 2015)
}

\begin{abstract}
We report on the first self-consistent numerical study of the feasibility of laser-driven relativistic pair shocks of prime interest for high-energy astrophysics. Using a QED-particle-in-cell code, we simulate the collective interaction between two counterstreaming electron-positron jets driven from solid foils by shortpulse ( $\sim 60 \mathrm{fs})$, high-energy $(\sim 100 \mathrm{~kJ})$ lasers. We show that the dissipation caused by self-induced, ultrastrong $\left(>10^{6} \mathrm{~T}\right)$ electromagnetic fluctuations is amplified by intense synchrotron emission, which enhances the magnetic confinement and compression of the colliding jets.
\end{abstract}

DOI: 10.1103/PhysRevLett.115.215003

PACS numbers: 52.27.Ep, 52.38.-r, 52.65.Rr, 52.72.+v

Collisionless turbulent shocks between energetic outflows may happen in various astrophysical environments (active galaxy nuclei, pulsar wind nebulae, supernovae remnants, etc.), where they are held responsible for the generation of nonthermal particles and radiation [1-3]. In the unmagnetized regime and for high enough velocities, first-principles particle-in-cell (PIC) simulations have demonstrated that the Weibel-filamentation instability [4-6] provides the magnetic turbulence needed for efficient dissipation and Fermi-type acceleration [7-9].

Along with theoretical progress, intense effort is ongoing worldwide to reproduce these phenomena in laser experiments [10]. The goal is to develop test beds for scenarios of shock formation and evolution. The most explored route relies on high-energy nanosecond lasers to trigger electronion shocks in counterstreaming nonrelativistic ablative flows [10-12]. Weibel-type ion filamentation has been recently observed using a few-kJ laser drive $[13,14]$, thus indicating that shock generation should be within the reach of National Ignition Facility (NIF)-class (>100 kJ) systems. Alternative configurations employing relativisticintensity lasers have also been proposed $[15,16]$.

The experimental study of relativistic electron-positron $\left(e^{-} e^{+}\right)$instabilities, which are likely to mediate the termination shocks of pulsar winds [17], appears more challenging owing to the difficulty of creating pair plasmas dense enough so that their typical dimensions largely exceed their relativistic skin depth [18]. Advances in this direction have led to record positron densities of $\sim 10^{16} \mathrm{~cm}^{-3}$ using mmthick high- $Z$ targets, either directly irradiated by intense $\left(\lesssim 10^{20} \mathrm{~W} \mathrm{~cm}^{-2}\right)$ picosecond lasers [19] or penetrated by wakefield-driven electron beams [20]. According to Ref. [19], laser energies of a few tens of $\mathrm{kJ}$ are required to produce pair plasmas prone to collisionless shock formation. In these experiments, positrons mainly originate from the Bethe-Heitler process, i.e., the interaction between bremsstrahlung photons and the Coulomb field of high- $Z$ nuclei. This emission occurs as fast electrons diffuse through the converter target, therefore over spatiotemporal scales much larger than the laser pulse.

Here, we explore an alternative concept that exploits the extreme fields envisioned via novel amplification techniques on large-scale NIF-class lasers [21]. Recent studies predict that laser intensities exceeding $10^{23} \mathrm{~W} \mathrm{~cm}^{-2}$ entail a new interaction regime characterized by intertwined collective and quantum electrodynamics (QED) effects [22-26]. For micrometric solid targets, the dominant QED processes are (i) nonlinear Compton emission of $\gamma$-ray photons by laser-accelerated electrons, and (ii) pair production by the multiphoton Breit-Wheeler process [27]. In contrast to the Bethe-Heitler-dominated regime, these mechanisms are mediated by the laser field and thus operate inside the irradiated region, thereby allowing much denser $e^{-} e^{+}$sources.

In this Letter, we present the first self-consistent numerical study of the interaction of two counterstreaming $e^{-} e^{+}$flows driven from thin solid foils by short-pulse ( $\sim 0 \mathrm{fs})$, highenergy $(\sim 2 \times 100 \mathrm{~kJ})$ lasers. Our QED-PIC simulation predicts the formation of neutral pair plasmas of density and size sufficient to trigger ultrafast $(<50 \mathrm{fs})$, Weibelinduced thermalization and compression through the buildup of a $\sim 2 \times 10^{6} \mathrm{~T}$ magnetostatic barrier. Remarkably, synchrotron radiation appears to strongly contribute to the energy dissipation and particle confinement. Although this long-term scenario does not yield a fully formed pair shock due to too short an interaction time, it opens exciting prospects for the laser probing of relativistic shocks in high-energy astrophysics.

Following Refs. [28,29], the PIC code CALDER [30] has been enriched with numerical models of nonlinear Compton radiation and Breit-Wheeler pair production [31]. Lowenergy photon emission is treated using Sokolov's radiation 
friction model with a quantum-corrected radiated power [32]. In the high-energy quantum regime, Monte Carlo schemes are used to describe both the discrete emission of photons and their annihilation into $e^{-} e^{+}$pairs.

The simulation setup consists of a pair of identical targets irradiated by two laser plane waves coming from both sides of the domain. The physical parameters are chosen so as to maximize the size to skin-depth ratio of the pair plasmas with a laser energy of about $100 \mathrm{~kJ}$. The linearly polarized laser pulses have a $1-\mu \mathrm{m}$ wavelength, a 64 -fs $\left(120 \omega_{0}^{-1}\right.$, where $\omega_{0}$ is the laser frequency) FWHM duration and a $8.9 \times 10^{23} \mathrm{~W} \mathrm{~cm}^{-2}$ peak intensity (i.e., a normalized field amplitude $\left.a_{0}=e E_{0} / m_{e} c \omega_{0}=800\right)$. Since optimum pair production occurs near the relativistic transparency threshold $\left(n_{e} \sim a_{0} n_{c}\right.$, where $n_{c} \simeq 1.1 \times 10^{21} \mathrm{~cm}^{-3}$ is the critical density) [24], the targets are taken to be fully ionized, solid $\mathrm{Al}$ foils of electron density $n_{e}=780 n_{c}$ and 5- $\mu$ m thickness. A $2-\mu \mathrm{m}$ density ramp is added on their front sides to mimic prepulse effects. They are placed symmetrically at a distance of $200 \mathrm{c} / \omega_{0}(32 \mu \mathrm{m})$ from the center of the domain. The latter has dimensions of $510 c / \omega_{0}(81 \mu \mathrm{m})$ in $x$ and $90 c / \omega_{0}$ $(14.5 \mu \mathrm{m})$ in $y$, with mesh size $\Delta x=\Delta y=0.03 c / \omega_{0}$. The boundary conditions are absorbing in $x$ and periodic in $y$ for both the fields and particles. Each cell initially contains 30 macroparticles per species, giving a total number of $3 \times 10^{8}$ macroparticles in the domain. The inflating number of particles and photons due to QED cascading [22] poses a severe computational burden, which restricts the simulation to a 2D geometry.

Laser-driven pair generation proceeds similarly to that described in Refs. [22,24], so we only outline its main features. Figure 1(a) shows that, at the end of the irradiation $\left(\omega_{0} t=555\right)$, a neutral $e^{-} e^{+}$jet has formed behind each target, with maximum density $n_{\max } \simeq 45 n_{c}$ at $\omega_{0} x / c \simeq 160$. The jet FWHM $\left(\tau_{j} \sim 80 \omega_{0}^{-1}\right)$ is somewhat shorter than the
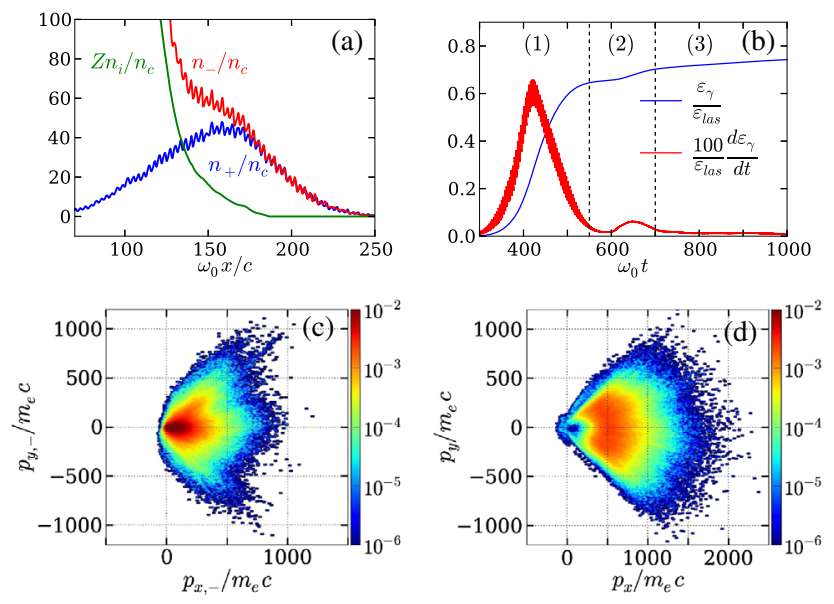

FIG. 1 (color online). (a) Transversely averaged particle densities at the rear of the left target prior to the collision $\left(\omega_{0} t=555\right)$. (b) Time evolution of the total radiated energy (blue) and power (red) normalized to the laser energy. (c),(d) Electron and positron $p_{x}-p_{y}$ phase spaces at the center of the pair plasma $\left(x=155 c / \omega_{0}\right)$ prior to the collision $\left(\omega_{0} t=555\right)$. laser duration owing to the rapid drop of the $e^{-} e^{+}$emission rate with decreasing laser intensity [33]. Closer to the target, the electron density exceeds the positron density so as to screen the slower expanding ions. The jet particles are characterized by broad, space-dependent momentum distributions, as partially illustrated in Figs. 1(c) and 1(d), which display the $p_{x}-p_{y}$ phase spaces near the density maximum $\left(\omega_{0} x / c \sim 155\right)$. The most energetic particles are located at the front $\left(\omega_{0} x / c \sim 250\right)$ with mean Lorentz factors $\left\langle\gamma_{-}\right\rangle \sim 550$ for the electrons (close to the ponderomotive scaling $\left\langle\gamma_{-}\right\rangle \sim \sqrt{1+a_{0}^{2} / 2}$ ) and $\left\langle\gamma_{+}\right\rangle \sim 1100$ for the positrons. The factor of $\sim 2$ between $\left\langle\gamma_{+}\right\rangle$and $\left\langle\gamma_{-}\right\rangle$ stems from the energy boost provided by the rear-side sheath potential set up by the (denser) fast electrons [24]. This forward-acceleration results in greater collimation of the positrons $\left(\left\langle\theta_{+}^{2}\right\rangle^{1 / 2} \sim 0.13\right)$ relative to the electrons $\left(\left\langle\theta_{-}^{2}\right\rangle^{1 / 2} \sim 0.6\right)$. The $e^{-} e^{+}$energies decrease deeper into the flow, reaching $\left\langle\gamma_{-}\right\rangle \sim 250$ and $\left\langle\gamma_{+}\right\rangle \sim 600$ around the density maximum. The electron cooling may result from the ponderomotive steepening of the irradiated surface [34].

Besides pair generation, the target as a whole is accelerated during the interaction. The fastest $\mathrm{Al}^{13+}$ ions originate from the target front: after being driven by the laser radiation pressure, they are further accelerated by the sheath field in the wake of the positrons, with maximum longitudinal momentum $p_{x} / m_{i} c \simeq 0.9$. The rear-side ions are slower $\left(p_{x} / m_{i} c \simeq 0.5\right.$ ) since they experience the sheath field only.

Overall, $65 \%$ of the laser energy is converted into highenergy radiation, $5 \%$ into positrons, $7 \%$ into fast electrons, and $10 \%$ into fast ions, yielding a total absorption fraction of $90 \%$. On the numerical side, $6 \times 10^{9}$ macrophotons are created during this phase.

The two $e^{-} e^{+}$jets make contact at the center of the domain $\left(\omega_{0} x / c=255\right)$ at $\omega_{0} t \approx 550$ (see movie in the Supplemental Material [35]). The collective interaction of pair jets has been previously simulated $[7,36]$, yet under simplified conditions of doubtful relevance for laser experiments. The present work is the first allowing for the self-consistent kinetic and radiative properties of the laser-induced pair beams.

Magnetic channels parallel to the beams initially develop in the interval $235 \lesssim \omega_{0} x / c \lesssim 280$, with an amplitude $B_{z} \simeq$ $150 m_{e} \omega_{0} / e \simeq 1.5 \times 10^{6} \mathrm{~T}$ and a transverse wavelength $\lambda \simeq 20 c / \omega_{0} \simeq 2 \pi c \sqrt{\gamma_{-}} / \omega_{p-}$, where $\omega_{p-}=\omega_{0} \sqrt{n_{-} / n_{c}}$ is the electron plasma frequency (assuming typical values $\gamma_{-}=250$ and $n_{-}=n_{\max } / 2$ for the electron Lorentz factor and density, respectively). Figures 2(a) and 2(b) show that these filaments, made of counterstreaming electrons and positrons, are magnetically pinched up to twice the jet initial density $\left(\sim 90 n_{c}\right)$. The time history of the field energies in the overlap region [Fig. 2(c)] confirms the essentially magnetic character of the instability: the magnetic energy exceeds the electric energy by about an order of magnitude and increases exponentially at a rate $\Gamma \simeq 0.24 \omega_{0}$. This value is consistent with the maximum filamentation growth rate ( $\Gamma_{\text {th }} \simeq 0.2 \omega_{0}$ at the wavelength $\left.\lambda_{\text {th }} \simeq 12 c / \omega_{0}\right)$ computed 

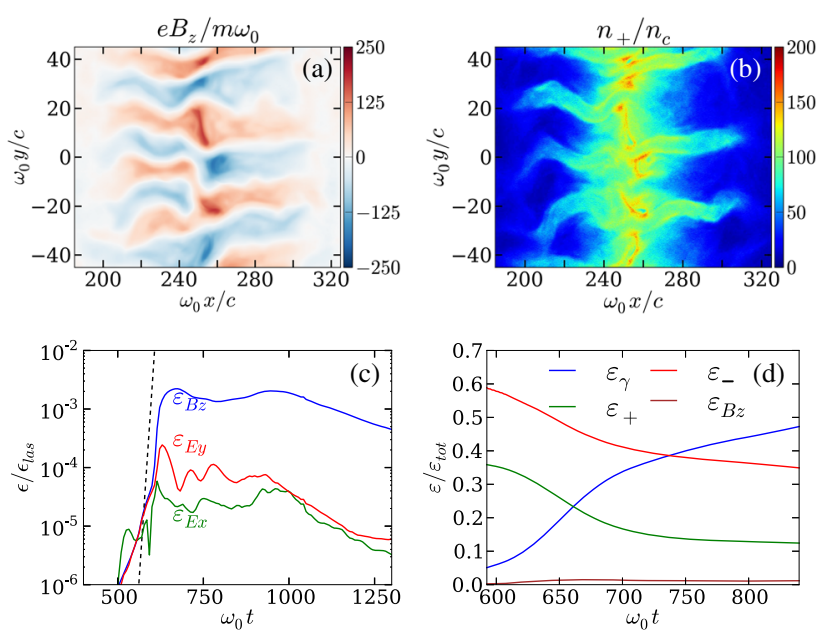

FIG. 2 (color online). (a),(b) Magnetic field (averaged over a laser cycle) in the overlap region at $\omega_{0} t=705$. (b) Positron density at $\omega_{0} t=705$. (c) Time evolution of the electromagnetic energies (normalized to the laser energy) integrated in the overlap region and comparison with the theoretical growth rate (dashed line). (d) Evolution of the particle $\left(\varepsilon_{ \pm}\right)$, photon $\left(\varepsilon_{\gamma}\right)$, and magnetic $\left(\varepsilon_{B_{z}}\right)$ energies (normalized to the total kinetic energy) in the collision region.

from a multiwaterbag model of the $p_{x}-p_{y}$ distribution [37]. Such a large growth rate $\left(\Gamma \tau_{j} \gg 1\right)$ ensures strong interaction between the jets during their overlap time. Figure 2(c) shows that the instability reaches saturation at $\omega_{0} t \simeq 680$. The saturated magnetic amplitude $B_{\text {sat }}$ and wavelength $\lambda_{\text {sat }}$ can be understood upon assuming complete current separation inside Alfvén-limited filaments [38]. Because of the lower relativistic inertia of the electrons, the half-width of the filaments should be close to the electron Larmor radius $\sim 2 m_{e} c \gamma_{-} / e B_{\text {sat }}$ (where the mean field in a filament is estimated to be $\left.B_{\text {sat }} / 2\right)$. Taking into account the $e^{-} e^{+}$ currents and a mean velocity $\left\langle v_{x}\right\rangle \sim c / 2$, one obtains the maximum field $e B_{\text {sat }} / m_{e} \omega_{0} \sim \sqrt{2 \gamma_{-} n_{-} / n_{c}} \sim 105$ with a wavelength $\lambda_{\text {sat }} \omega_{0} / c \sim 4 \sqrt{2 \gamma_{-} n_{c} / n_{-}} \sim 19$, in agreement with the simulation.

By the end of the collision $\left(\omega_{0} t=705\right)$, the magnetic channels have extended longitudinally over $200 \lesssim$ $\omega_{0} x / c \lesssim 310$, with a peak amplitude $B_{z} \simeq 230 m_{e} \omega_{0} / e$ [Fig. 2(a)]. Kinklike oscillations have destroyed the coherence of the filaments around the collision plane, which contributes to the particle thermalization [39]. Figures 3(a) and 3(b) indeed show essentially isotropized $e^{-} e^{+}$populations in the central region by $\omega_{0} t=690$. Also, the corresponding $\gamma$ distributions are well reproduced by MaxwellJüttner functions $f_{\mathrm{MJ}}(\gamma) \propto \gamma e^{-m_{e} c^{2} \gamma / T}$ with electron and positron temperatures $T_{-} \simeq 63 m_{e} c^{2}$ and $T_{+} \simeq 85 m_{e} c^{2}$, respectively [Fig. 3(c)].

That the inferred temperatures are significantly lower than $m_{e} c^{2}\left\langle\gamma_{ \pm}\right\rangle / 2$ (the value expected for complete thermalization) stems from strong radiative losses during, or shortly after the turbulence buildup. The radiated energy and power are plotted versus time in Fig. 1(b). The first,
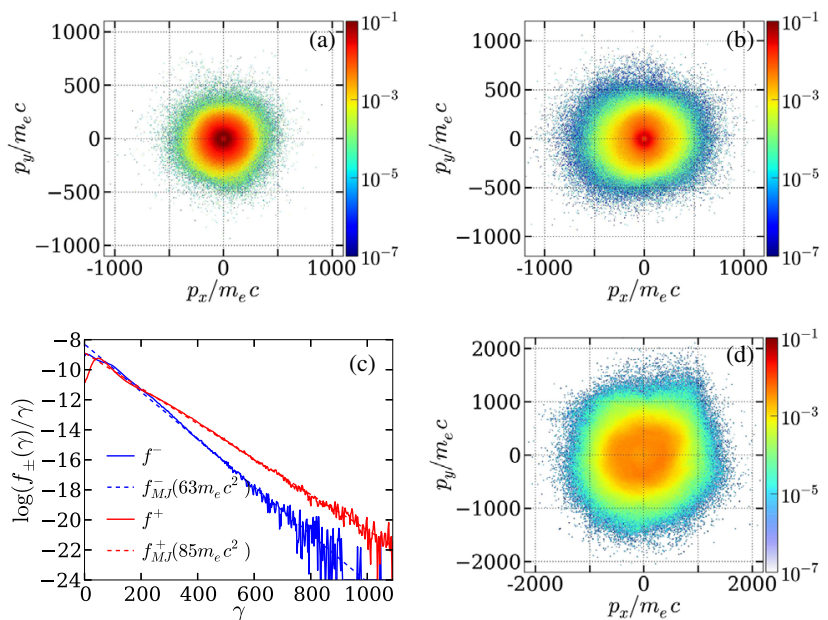

FIG. 3 (color online). (a) Electron and (b) positron $p_{x}-p_{y}$ phase spaces at the center of the domain at the time of maximum compression $\left(\omega_{0} t=690\right)$. (c) Corresponding electron (red) and positron (blue) $\gamma$ distributions and comparison with the bestfitting Maxwell-Jüttner distributions (dashed lines). (d) Positron $p_{x}-p_{y}$ phase space without radiation losses.

strongly radiating phase $\left(\omega_{0} t<550\right)$ corresponds to the laser-target interaction. The second, lesser-emitting phase $\left(550<\omega_{0} t<705\right)$ is associated with the pair plasma collision. About $60 \%$ of the jet energy is radiated away in less than $100 \omega_{0}^{-1}$ due to synchrotron emission in the magnetic turbulence [comprising 3\% of the jet energy, Fig. 2(d)]. The spectrum peaks around $300 \mathrm{keV}$ and extends from a few $\mathrm{keV}$ to a few 100s of $\mathrm{MeV}$ (not shown). The above temperatures then prove consistent with almost complete thermalization of the jets when allowing for radiative losses. This ultrafast radiative cooling follows from the combination of extreme kinetic energies and quasistatic fields specific to the laser-solid configuration. Its time scale can be estimated from the radiated power [27] as $\tau_{c} \sim 3 \times 10^{7}\left(\gamma^{2} n / n_{c}\right)^{-1} \mathrm{fs} \sim 16 \mathrm{fs}$. To better assess its impact upon the two-stream interaction, we have run the same simulation with synchrotron emission switched off during the collision. While this simulation predicts a similar evolution of the field energies, it leads to major changes in the phase space. Figure 3(d) shows that the positrons, albeit significantly isotropized, are much less thermalized: their momentum distribution presents a broad plateau up to $|p| \sim 500 m_{e} c$, with maximum momenta increased by a factor $\sim 2$.

Significant compression ensues from the slowing down and isotropization of the jets. In the radiative case [Fig. 4(a)], a peak positron density of $128 n_{c}$ is reached at $\omega_{0} t \simeq 685$ in the overlap zone, corresponding to a compression ratio $R \simeq 2.85$ relative to the unperturbed jet density $\left(\simeq 45 n_{c}\right)$. This compression, however, is not sustained at later times due to the decreasing power flux carried by the jets. Neglecting radiation [Fig. 4(b)] entails higher particle losses, a weaker compression $(R \simeq 2.4)$, and a much shorter confinement time $\left(\simeq 100 \omega_{0}^{-1}\right)$ than in the radiative case, where a dense structure $\left(\simeq 70 n_{c}\right)$ survives over a time $\gtrsim 500 \omega_{0}^{-1}$. 

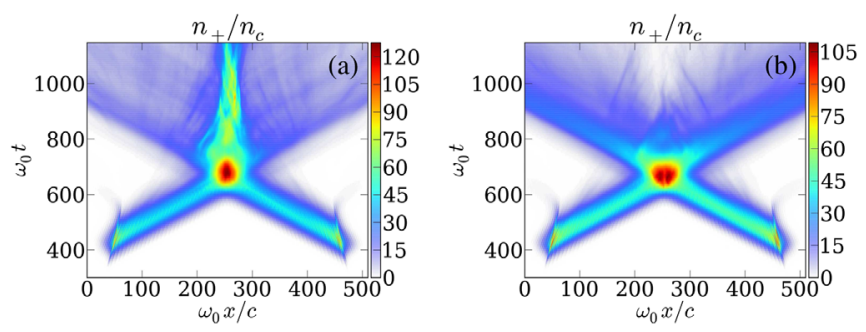

FIG. 4 (color online). Space-time evolution of the $y$-averaged positron density with (a) and without (b) radiation losses.

To examine the effects of radiative cooling upon a Weibelinstability-mediated shock, we have simulated the collision of two pair plasmas injected from the $x$ boundaries with density $\left(n_{\max }=50 n_{c}\right)$ and $x$ momentum $\left(p_{x} / m_{e} c= \pm 500\right)$ close to those of the laser-driven plasmas. Initially, each flow occupies half the $6000 \mathrm{c} / \omega_{0}$-long box and has a uniform density profile preceded by a $80 c / \omega_{0}$-long ramp. Figure 5(a) shows that the compression of the central region attains the value associated with a nonradiative, $2 \mathrm{D}$ relativistic shock $\left(R \simeq 3\right.$ ) by $\omega_{0} t \simeq 110$ (the shock forms at $\omega_{0} t \simeq 150$ in a similar nonradiative simulation), but keeps on increasing later on as the shock front steadily decelerates: at $\omega_{0} t=1200, R \simeq 9$ and the front velocity has dropped to $v_{\text {sh }} \simeq 0.12 c$. The shock nonstationarity is accompanied by continuous cooling of the compressed plasma, the mean positron energy decreasing from $\gamma_{+} \simeq 180$ at $\omega_{0} t=200$ down to $\gamma_{+} \simeq 85$ at $\omega_{0} t=1200$ [Fig. 5(b)]. A simulation run with $n_{\max }=12.5$ shows a similar evolution, with a compression rising to $R \simeq 5.5$ over the same time span. Pair annihilation and Compton scattering of energetic photons are expected to be negligible on the considered spatiotemporal scales. The $B_{z}$-field map displayed at $\omega_{0} t=400$ in the top panel of Fig. 5(c) exhibits a transition layer of length $\sim 15 \sqrt{\gamma_{+}} c / \omega_{p}$, across which the filaments formed in the upstream precursor region decay into clumps, and the plasma
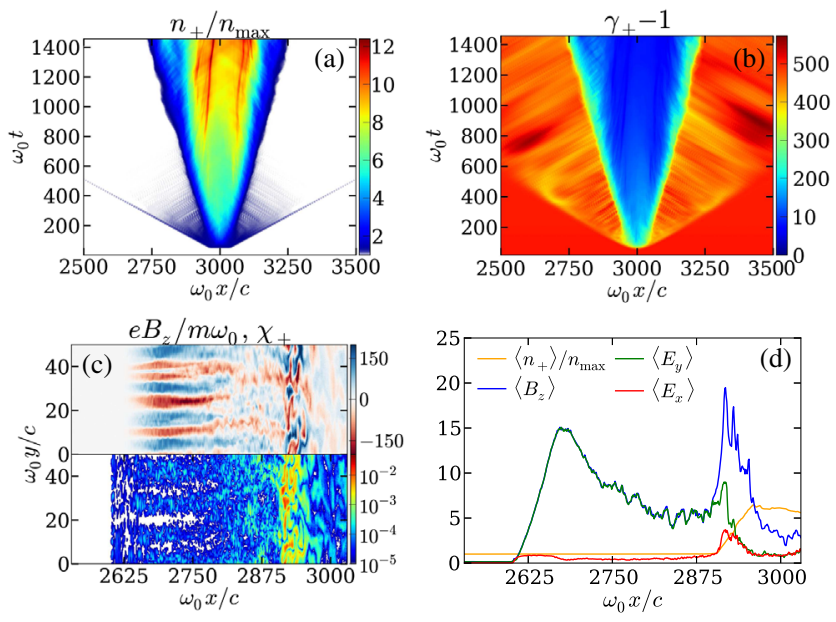

FIG. 5 (color online). Space-time evolution of the $y$-averaged positron (a) compression ratio and (b) Lorentz factor. (c) Magnetic field $B_{z}$ (top) and quantum parameter $\chi_{+}$(bottom) at $\omega_{0} t=400$. (d) $y$-averaged profiles of $\left|E_{x}\right|,\left|E_{y}\right|, c\left|B_{z}\right|$ (normalized to $m_{e} c \omega_{0} / e$ ) and $n_{+} / n_{\max }$ at $\omega_{0} t=400$. compression and cooling take place [Figs. 5(b) and 5(d)]. To elucidate the radiation process, the bottom panel of Fig. 5(c) plots the spatial distribution of the dimensionless parameter $\chi_{+} \simeq e \hbar \gamma_{+}\left(E_{y}-c B_{z}\right) / m_{e}^{2} c^{3}$, noting that the radiated power scales as $P_{\text {rad }} \propto \chi_{+}^{2}$ [27]. Very low $\chi_{+}$values $\left(<10^{-3}\right)$ are reached in the precursor region due to the motional field $E_{y} \sim c B_{z}$ induced in the filaments, yielding negligible radiation losses. By contrast, $\left|B_{z}\right| \gg\left|E_{y}\right| / c$ in the transition layer, so that $\chi_{+}$abruptly rises up to $\sim 5 \times 10^{-2}$ [Fig. 5(d)]. Most of the radiation is therefore emitted across the turbulent layer. As the plasma cools down, magnetic trapping effects are strengthened, which weakens the turbulence damping [40]. The magnetic layer consequently thickens with time, leading to enhanced radiative losses and an increasingly dense and cold downstream plasma [Figs. 5(a) and 5(b)]. Importantly, these losses are strong enough to quench the Fermi acceleration process [41].

Our idealized plane-wave setup involves a total laser energy of $\sim 2 \times 120 \mathrm{~kJ}$, assuming a focal spot $D_{0}=90 \mathrm{c} / \omega_{0}$ for each pulse. In a realistic 3D geometry, due to the radial expansion of the particles, the on-axis jet density should weakly decrease up to a distance $x_{d} \sim D_{0} / 2 \sqrt{2\left\langle\theta^{2}\right\rangle}$ from the target. Further away, it should drop as $n(x) \sim$ $n_{\max }\left(x_{d} / x\right)^{2}$ due to an increasing transverse size $D(x) \sim$ $2 \sqrt{2\left\langle\theta^{2}\right\rangle} x$ [42]. Estimating the effective dispersion angle as the $\gamma$-weighted average of the $e^{-} e^{+}$values, one obtains $\left\langle\theta^{2}\right\rangle^{1 / 2} \sim 0.3$ and $x_{d} \sim 100 c / \omega_{0}$. The experimental reproduction of our integrated simulation with a similar laser drive would then require a target separation below $2 x_{d} \sim 200 c / \omega_{0}$, yet larger than the shock width $\sim 100 c / \omega_{0}$. From Fig. 5(a), the clear observation of a propagating shock implies an interaction time of $\sim 200-300 \omega_{0}^{-1}$, that is, 3-4 times larger than obtained with our laser parameters. For an unchanged intensity, this would correspond to a $\sim 1 \mathrm{MJ}$ total laser energy.

In conclusion, we have presented the first integrated simulation gauging the potential of future extreme-intensity lasers to trigger $e^{-} e^{+}$pair shocks. We have shown that the collective interaction of two pair jets created from solid targets by $60 \mathrm{fs}, 120 \mathrm{~kJ}$ lasers is able to thermalize a significant part of the bulk jet energy, yet failing to spawn a fully developed, propagating shock due to too short an interaction time. An important finding is that the synchrotron emission induced in the magnetic turbulence $\left(>10^{6} \mathrm{~T}\right)$ dissipates $\sim 60 \%$ of the kinetic energy in a few tens of fs. These losses speed up the thermalization of the jets, and enhance their magnetic confinement and compression. This novel, laser-specific interaction regime contrasts with the standard scenario of astrophysical pair shocks, where synchrotron emission mainly occurs deep into the downstream region, well after shock formation. Our results indicate that longer-duration ( $200 \mathrm{fs})$, higher-energy $(\sim \mathrm{MJ})$ lasers are required for the creation of fully formed pair shocks through nonlinear Compton or Breit-Wheeler processes. 
The authors acknowledge support from the Agence Nationale de la recherche (LABEX PALM-ANR-10LABX-39, ANR SILAMPA) and from GENCI for HPC resources at TGCC/CCRT (DARI Project No. x2013052707). Thanks are due to S. V. Bulanov, R. Capdessus, $\mathrm{X}$. Davoine, R. Duclous and M. Touati for valuable discussions.

*mathieu.lobet@cea.fr †laurent.gremillet@cea.fr

[1] M. A. Malkov and L. O'C Drury, Rep. Prog. Phys. 64, 429 (2001).

[2] T. Piran, Rev. Mod. Phys. 76, 1143 (2005).

[3] M. Lemoine, G. Pelletier, and B. Revenu, Astrophys. J. Lett. 645, L129 (2006).

[4] M. V. Medvedev and A. Loeb, Astrophys. J. Lett. 526, 697 (1999).

[5] A. Achterberg and J. Wiersma, Astron. Astrophys. 475, 1 (2007); A. Achterberg, J. Wiersma, and C. A. Norman, Astron. Astrophys. 475, 19 (2007).

[6] M. Lemoine and G. Pelletier, Mon. Not. R. Astron. Soc. 417, 1148 (2011).

[7] T. N. Kato, Astrophys. J. 668, 974 (2007); T. N. Kato and H. Takabe, Astrophys. J. Lett. 681, L93 (2008).

[8] A. Spitkovsky, Astrophys. J. Lett. 682, L5 (2008).

[9] A. Stockem, F. Fiuza, A. Bret, R. A. Fonseca, and L. O. Silva, Sci. Rep. 4, 3934 (2014).

[10] R. P. Drake and G. Gregori, Astrophys. J. 749, 171 (2012).

[11] Y. Kuramitsu et al., Phys. Rev. Lett. 106, 175002 (2011).

[12] H.-S. Park et al., High Energy Density Phys. 8, 38 (2012).

[13] W. Fox, G. Fiksel, A. Bhattacharjee, P.-Y. Chang, K. Germaschewski, S. X. Hu, and P. M. Nilson, Phys. Rev. Lett. 111, 225002 (2013).

[14] C. M. Huntington, F. Fiuza, J. S. Ross, A. B. Zylstra, R. P. Drake, D. H. Froula, G. Gregori, N. L. Kugland, C. C. Kuranz, M. C. Levy, C. K. Li, J. Meinecke, T. Morita, R. Petrasso, C. Plechaty, B. A. Remington, D. D. Ryutov, Y. Sakawa, A. Spitkovsky, H. Takabe, and H.-S. Park, Nat. Phys. 11, 173 (2015).

[15] F. Fiuza, R. A. Fonseca, J. Tonge, W. B. Mori, and L. O. Silva, Phys. Rev. Lett. 108, 235004 (2012).

[16] S. P. Davis, R. Capdessus, E. d'Humières, S. Jequier, I. Andriyash, and V.T. Tikhonchuk, High Energy Density Phys. 9, 231 (2013).

[17] A. M. Bykov and R. A. Treumann, Astron. Astrophys. Rev. 19, 42 (2011).

[18] E. Liang, High Energy Density Phys. 9, 425 (2013).

[19] H. Chen, S. C. Wilks, J. D. Bonlie, E. P. Liang, J. Myatt, D. F. Price, D. D. Meyerhofer, and P. Beiersdorfer, Phys. Rev. Lett. 102, 105001 (2009); H. Chen, F. Fiuza, A. Link, A. Hazi, M. Hill, D. Hoarty, S. James, S. Kerr, D. D. Meyerhofer, J. Myatt, J. Park, Y. Sentoku, and G. J. Williams, Phys. Rev. Lett. 114, 215001 (2015).

[20] G. Sarri, K. Poder, J. Cole, W. Schumaker, A. Di Piazza, B. Reville, T. Dzelzainis, D. Doria, L. Gizzi, G. Grittani, S. Kar, C. Keitel, K. Krushelnick, S. Kuschel, Z. Mangles, Z. Najmudin, N. Shukla, L. Silva, D. Symes, A. Thomas, M. Vargas, J. Vieira, and M. Zepf, Nat. Commun. 6, 6747 (2015).
[21] G. Mourou and T. Tajima, Science 331, 41 (2011); G. A. Mourou, N. J. Fisch, V. M. Malkin, Z. Toroker, E. A. Khazanov, A. M. Sergeev, T. Tajima, and B. Le Garrec, Opt. Commun. 285, 720 (2012); G. Mourou and T. Tajima, Eur. Phys. J. Spec. Top. 223, 979 (2014).

[22] E. N. Nerush, I. Y. Kostyukov, A. M. Fedotov, N. B. Narozhny, N. V. Elkina, and H. Ruhl, Phys. Rev. Lett. 106, 035001 (2011).

[23] A. Di Piazza, C. Müller, K. Z. Hatsagortsyan, and C. H. Keitel, Rev. Mod. Phys. 84, 1177 (2012).

[24] C. P. Ridgers, C. S. Brady, R. Duclous, J. G. Kirk, K. Bennett, T. D. Arber, A. P. L. Robinson, and A. R. Bell, Phys. Rev. Lett. 108, 165006 (2012); C. P. Ridgers, C. S. Brady, R. Duclous, J. G. Kirk, K. Bennett, T. D. Arber, and A. R. Bell, Phys. Plasmas 20, 056701 (2013).

[25] C. S. Brady, C. P. Ridgers, T. D. Arber, and A. R. Bell, Phys. Plasmas 21, 033108 (2014).

[26] L. L. Ji, A. Pukhov, E. N. Nerush, I. Y. Kostyukov, B. F. Shen, and K. U. Akli, Phys. Plasmas 21, 023109 (2014).

[27] J. G. Kirk, A. R. Bell, and I. Arka, Plasma Phys. Controlled Fusion 51, 085008 (2009).

[28] R. Duclous, J. G. Kirk, and A. R. Bell, Plasma Phys. Controlled Fusion 53, 015009 (2011).

[29] N. V. Elkina, A. M. Fedotov, I. Y. Kostyukov, M. V. Legkov, N. B. Narozhny, E. N. Nerush, and H. Ruhl, Phys. Rev. ST Accel. Beams 14, 054401 (2011).

[30] E. Lefebvre et al., Nucl. Fusion 43, 629 (2003).

[31] M. Lobet, E. d'Humières, M. Grech, C. Ruyer, X. Davoine, and L. Gremillet, arXiv:1311.1107.

[32] I. V. Sokolov, N. M. Naumova, J. A. Nees, G. A. Mourou, and V. P. Yanovsky, Phys. Plasmas 16, 093115 (2009).

[33] A. R. Bell and J. G. Kirk, Phys. Rev. Lett. 101, 200403 (2008).

[34] B. Chrisman, Y. Sentoku, and A. J. Kemp, Phys. Plasmas 15, 056309 (2008).

[35] See Supplemental Material at http://link.aps.org/ supplemental/10.1103/PhysRevLett.115.215003 for Twodimensional CALDER PIC-QED simulation of the pair plasma generation and collision induced by two laser planes waves of $8.9 \times 10^{23} \mathrm{~W} \mathrm{~cm}^{-2}$ intensity and 64-fs duration, interacting with two 5- $\mu \mathrm{m} \mathrm{Al}$ targets. Left column, from top down: ion density, electron density, positron density, $y$-averaged particle densities and electric field $\left|E_{y}\right|$ (see legend). Right column, from top down: magnetic field $B_{z}$ averaged over a laser period, positron and electron $x-p_{x}$ phase spaces, electromagnetic energies (normalized to the total laser energy) integrated in the overlap region $185 \leq x \leq 325 c / \omega_{0}$ (bottom, left) and total radiated energy normalized to the total laser energy (bottom, right).

[36] A. Spitkovsky, Astrophys. J. Lett. 673, L39 (2008).

[37] C. Ruyer, L. Gremillet, and G. Bonnaud, Phys. Plasmas 22, 082107 (2015).

[38] T. N. Kato, Phys. Plasmas 12, 080705 (2005).

[39] M. Milosavljević and E. Nakar, Astrophys. J. 641, 978 (2006).

[40] P. Chang, A. Spitkovsky, and J. Arons, Astrophys. J. 674, 378 (2008).

[41] M. V. Medvedev and A. Spitkovsky, Astrophys. J. 700, 956 (2009).

[42] A. Debayle, J. J. Honrubia, E. d'Humières, and V. T. Tikhonchuk, Phys. Rev. E 82, 036405 (2010). 